\title{
Machine learning universal bosonic functionals
}

\author{
Jonathan Schmidt $\odot,{ }^{1}$ Matteo Fadel $\odot,{ }^{2}$ and Carlos L. Benavides-Riveros $\oplus^{3,4, *}$ \\ ${ }^{1}$ Institut für Physik, Martin-Luther-Universität Halle-Wittenberg, 06120 Halle (Saale), Germany \\ ${ }^{2}$ Department of Physics, University of Basel, Klingelbergstrasse 82, 4056 Basel, Switzerland \\ ${ }^{3}$ Max Planck Institute for the Physics of Complex Systems, Nöthnitzer Straße 38, 01187 Dresden, Germany \\ ${ }^{4}$ NR-ISM, Division of Ultrafast Processes in Materials (FLASHit), Area della Ricerca di Roma 1, Via Salaria Km 29.3, \\ I-00016 Monterotondo Scalo, Italy
}

(Received 31 March 2021; accepted 25 August 2021; published 13 September 2021)

\begin{abstract}
The one-body reduced density matrix $\gamma$ plays a fundamental role in describing and predicting quantum features of bosonic systems, such as Bose-Einstein condensation. The recently proposed reduced density matrix functional theory for bosonic ground states establishes the existence of a universal functional $\mathcal{F}[\gamma]$ that recovers quantum correlations exactly. Based on a decomposition of $\gamma$, we have developed a method to design reliable approximations for such universal functionals: Our results suggest that for translational invariant systems the constrained search approach of functional theories can be transformed into an unconstrained problem through a parametrization of a Euclidian space. This simplification of the search approach allows us to use standard machine learning methods to perform a quite efficient computation of both $\mathcal{F}[\gamma]$ and its functional derivative. For the Bose-Hubbard model, we present a comparison between our approach and the quantum Monte Carlo method.
\end{abstract}

DOI: 10.1103/PhysRevResearch.3.L032063

In 1964, Hohenberg and Kohn proved the existence of a universal functional $\mathcal{F}[\rho]$ of the particle density $\rho$ that captures the exact electronic contribution to the groundstate energy of a system of interacting electrons [1]. Due to a remarkable balance of accuracy and computational cost, first-principles modeling of electronic systems based on the respective density functional theory (DFT) is nowadays a well-established daily practice, with great impact in the fields of materials science, quantum chemistry, and condensed matter [2]. For bosonic systems, however, a fully first-principles description has been elusive. This is due in part to the unsuitability of the particle density to describe fundamental bosonic features as orbital occupations, mode entanglement, or nondiagonal order, which are important for predicting and describing bosonic condensation. As a result, the theoretical treatment of interacting bosonic systems mainly relies on exact diagonalization techniques, which are restricted to a few tens of orbitals [3-5], or mean-field theories that are particularly suitable for dilute ultracold gases [6-8]. The quantum Monte Carlo (QMC) method is known to be a powerful family of techniques for computing ground-state properties but is still restricted due to the fermion sign problem. While bosonic systems do not suffer such a sign problem, the QMC method

\footnotetext{
*carlosbe@pks.mpg.de
}

Published by the American Physical Society under the terms of the Creative Commons Attribution 4.0 International license. Further distribution of this work must maintain attribution to the author(s) and the published article's title, journal citation, and DOI. Open access publication funded by the Max Planck Society. cannot be applied to, e.g., frustrated quantum spin systems [9]. Nowadays, a renewed interest in the $a b$ initio description of many-body systems has been motivated by the successful application of artificial neural networks to both fermionic and bosonic problems $[10,11]$.

Since the parameters of correlated bosonic systems (ultracold gases, in particular) can be tuned with a high degree of control, they are powerful platforms to study a wide range of model Hamiltonians, ranging from Hubbard models to bosonic antiferromagnets $[12,13]$. They have also become an active research field in the context of quantum simulations [14-16] and even quantum foundations [17-20]. Such a need to describe quantum correlations of bosonic systems efficiently has motivated quite recently the putting forward of a physical theory for interacting bosonic systems [21,22]. Based on a generalization of the Hohenberg-Kohn theorem [23,24], this reduced density matrix functional theory (RDMFT) for bosons establishes the existence of a universal functional $\mathcal{F}_{W}[\gamma]$ of the one-body reduced density matrix (1RDM): $\gamma \equiv$ $N \operatorname{Tr}_{N-1}[\Gamma]$, obtained from the $N$-boson density operator $\Gamma$ by integrating out all but one boson, and the two-particle interaction $\hat{W}$. Since the $1 \mathrm{RDM}$ is the natural variable of the theory, RDMFT is particularly well suited for the accurate description of Bose-Einstein condensates (BECs), strongly correlated bosonic systems, or fragmented BECs $[25,26]$. Furthermore, the information contained in the spectra of the 1RDM can also be sufficient to investigate multipartite quantum correlations in those systems [27-31].

Although RDMFT holds the promise of abandoning the complex $N$-particle wave function as the central object, it does not trivialize the ground-state problem. In fact, the fundamental challenge is to provide reliable approximations 
to the universal interaction functional $\mathcal{F}_{W}[\gamma]$. Yet, while the Hohenberg-Kohn-type foundational theorem of RDMFT shows the existence of a universal functional, it does not give any indication of its concrete form. For DFT, the solution to this problem is given in the form of large classes of approximate functionals, hierarchically organized in the so-called Jacob's ladder. In recent years, the number of such approximations has significantly increased thanks to machine learning [32-37] and reduced density matrix [38] approaches.

Our work succeeds in providing a strategy for computing approximations for $\mathcal{F}_{W}[\gamma]$. In this Research Letter we (i) provide an efficient method to capture the essential features of universal functionals for boson lattices, (ii) show how the constrained search approach associated with it can be simplified in the form of an unconstrained problem, and (iii) implement this approach in a standard machine learning library to compute $\mathcal{F}_{W}[\gamma]$, its derivative, and the ground-state energy. We shall for simplicity describe our method for the Bose-Hubbard model, but the same results apply to any type of interactions for systems with translational symmetry.

Universal bosonic functionals. In this Research Letter we consider Hamiltonians of the form

$$
\hat{H}_{W}(\hat{h}) \equiv \hat{h}+\hat{W},
$$

with a one-particle term $\hat{h}=\hat{t}+\hat{v}$, containing the kinetic energy and the external potential terms, and the two-particle interaction $\hat{W}$. The ground-state energy and 1RDM follow for any choice of the one-particle Hamiltonian $h$ from the minimization of the total energy functional $\mathcal{E}_{h}[\gamma]=\operatorname{Tr}[h \gamma]+$ $\mathcal{F}_{W}[\gamma]$. The functional $\mathcal{F}_{W}[\gamma]$ is universal in the sense that it depends only on the fixed interparticle interaction $W$ and not on the one-particle Hamiltonian $\hat{h}$. Hence determining the functional $\mathcal{F}_{W}[\gamma]$ would in principle entail the simultaneous solution of the universal correlation part of the ground-state problem for any Hamiltonian $H_{W}(h)$. By writing the groundstate energy as $E(h) \equiv \min _{\Gamma} \operatorname{Tr}_{N}\left[H_{W}(h) \Gamma\right]$, and using the fact that the expectation value of $h$ is determined by $\gamma$, one can replace the functional $\mathcal{F}_{W}[\gamma]$ by the well-known constrained search approach [39]:

$$
\mathcal{F}_{W}[\gamma]=\min _{\Gamma \mapsto \gamma} \operatorname{Tr}_{N}[W \Gamma],
$$

where $\Gamma \mapsto \gamma$ indicates that the minimization is carried out over all $\Gamma$ whose 1 RDM is $\gamma$. The main challenge of this approach is that the set of $\Gamma$ such that $\Gamma \mapsto \gamma$ is in general extremely complex to characterize, and so far only partial results are known for quasiextremal, two-particle, or translational invariant fermionic systems [40-44]. Even in the extremely popular DFT, the constrained search over manybody wave functions integrating to the same electronic density (i.e., $\Psi \rightarrow \rho$ ) is rarely explicitly carried out [45].

To shed some light on the problem, let us represent $\gamma$, the 1 RDM of an $N$-boson real wave function $|\Psi\rangle$, with respect to a set of creation and annihilation operators

$$
\gamma_{i j}=\left\langle\Psi\left|\hat{b}_{i}^{\dagger} \hat{b}_{j}\right| \Psi\right\rangle
$$

and assume that the dimension of the one-particle Hilbert space is $M$. Let us also define $M(N-1)$-particle wave functions $\left|\Phi_{j}\right\rangle \equiv \hat{b}_{j}|\Psi\rangle$, which satisfy by definition the condition

$$
\left\langle\Phi_{i} \mid \Phi_{j}\right\rangle=\gamma_{i j}
$$

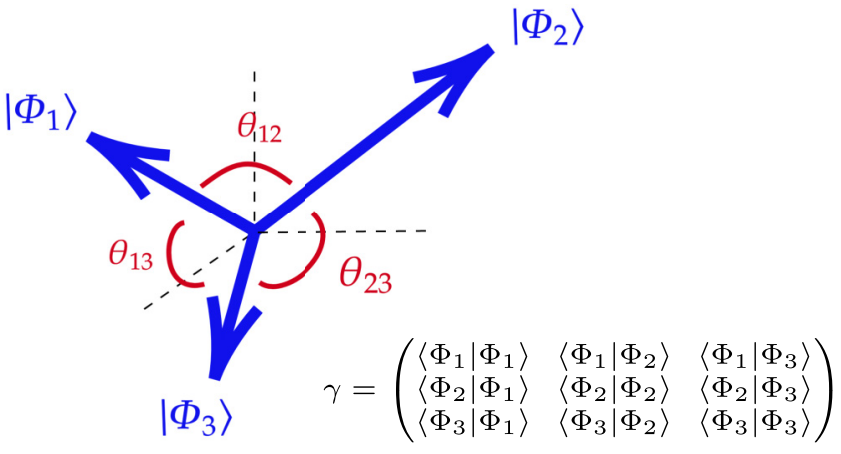

FIG. 1. Representation of three wave functions in the Hilbert space of $N-1$ particles giving place to a 1RDM $\gamma$. While the magnitude of the vectors is $\left\|\Phi_{i}\right\|^{2}=\gamma_{i i}$, the angles they form satisfy $\left\langle\Phi_{i} \mid \Phi_{j}\right\rangle=\sqrt{\gamma_{i i} \gamma_{j j}} \cos \left(\theta_{i j}\right)$.

The meaning of these non-normalized wave functions is clear: While their magnitude equals the diagonal entries of $\gamma$ (i.e., $\left\langle\Phi_{j} \mid \Phi_{j}\right\rangle=\gamma_{j j}$ ), the angles they form correspond to the nondiagonal entries of $\gamma$. Indeed, since $\left\langle\Phi_{i} \mid \Phi_{j}\right\rangle=$ $\left\|\Phi_{i}\right\|\left\|\Phi_{j}\right\| \cos \left(\theta_{i j}\right)=\sqrt{\gamma_{i i} \gamma_{j j}} \cos \left(\theta_{i j}\right)$, we have

$$
\cos \left(\theta_{i j}\right)=\frac{\gamma_{i j}}{\sqrt{\gamma_{i i} \gamma_{j j}}} \text {. }
$$

The bound of the nondiagonal entries, $\left|\gamma_{i j}\right|^{2} \leqslant \gamma_{i i} \gamma_{j j}$, is the Cauchy-Schwarz inequality for operators and is known to be a representability condition for $\gamma$ [46]. The condition $\sum_{j} \hat{b}_{j}^{\dagger}\left|\Phi_{j}\right\rangle=N|\Psi\rangle$ suggests that the minimizer of the minimization (2) can be written as a set of $M$ vectors in the Hilbert space $\mathcal{H}_{N-1}$ of $N-1$ particles, such that their angles and magnitudes are determined by Eq. (4) (see Fig. 1). We now exploit this first insight to explicitly carry out the constrained search approach and find the universal functional of the Bose-Hubbard model, a workhorse in the context of ultracold bosonic atoms [47].

Bose-Hubbard model. The Hamiltonian of the BoseHubbard model reads

$$
H=-t \sum_{\langle i j\rangle} \hat{b}_{i}^{\dagger} \hat{b}_{j}+\frac{U}{2} \sum_{j=1}^{M} \hat{n}_{j}\left(\hat{n}_{j}-1\right),
$$

where the operator $\hat{b}_{j}^{\dagger}\left(\hat{b}_{j}\right)$ creates (annihilates) a boson on site $j$, and $\hat{n}_{j}$ is the corresponding number operator. The first term in Eq. (6) describes the hopping between two sites, while the second one is the interacting term $\hat{W}=\frac{U}{2} \sum_{j} \hat{n}_{j}\left(\hat{n}_{j}-1\right)$. Since the problem is determined by $N$ spinless bosons and $M$ sites, we write for the functional $\mathcal{F}_{N, M}[\gamma]$. For a given $\gamma$, let us take the minimizer of the functional (2) and call it $\left|\Psi_{\gamma}\right\rangle \in \mathcal{H}_{N}$, the $N$-particle Hilbert space. Using the prescription discussed above, let us define $M(N-1)$-particle wave functions $\left|\Phi_{\gamma, j}\right\rangle \equiv \hat{b}_{j}\left|\Psi_{\gamma}\right\rangle \in \mathcal{H}_{N-1}$, which satisfy by definition the condition (4). Due to the translational invariance of the Bose-Hubbard Hamiltonian (6), these wave functions are all normalized to the filling factor, namely, $\left\langle\Phi_{\gamma, i} \mid \Phi_{\gamma, i}\right\rangle=N / M$. The functional is given by $\mathcal{F}_{N, M}[\gamma]=\sum_{i}\left\langle\Phi_{\gamma, i}\left|\hat{n}_{i}\right| \Phi_{\gamma, i}\right\rangle$, using $\hat{n}_{i}\left(\hat{n}_{i}-1\right)=\hat{b}_{i}^{\dagger} \hat{n}_{i} \hat{b}_{i}$. As shown in the supporting information [48], any rotation of the states $\left|\Phi_{\gamma, i}\right\rangle$ in the subspace spanned by themselves, $\mathcal{G}_{\gamma}=$ 
$\operatorname{span}\left\{\left|\Phi_{\gamma, 1}\right\rangle, \ldots,\left|\Phi_{\gamma, M}\right\rangle\right\}$, will give an energy greater than or equal to the energy $\mathcal{F}_{N, M}[\gamma]$. As a consequence, we rewrite the constrained search approach in Eq. (2) as

$$
\mathcal{F}_{N, M}[\gamma]=\min _{\left\{\Phi_{i}\right\} \in \mathcal{G}_{\gamma}} \sum_{i}\left\langle\Phi_{i}\left|\hat{n}_{i}\right| \Phi_{i}\right\rangle,
$$

subject to $\left\langle\Phi_{i} \mid \Phi_{i}\right\rangle=N / M$ and $\left\langle\Phi_{i} \mid \Phi_{j}\right\rangle=\gamma_{i j}$. This indicates that the constraint in Eq. (2) can be transferred to the subspace $\mathcal{G}_{\gamma}$. As we will see below, this result leads to a quite efficient optimization problem for the functional.

Exact functional of the dimer. As a first illustration of this approach, let us take the simple case of the Bose-Hubbard dimer with two particles $(N=M=$ 2). The states of the Hilbert space can be written as two occupations: $\left|n_{L}, n_{R}\right\rangle$. For the one-boson Hilbert space we choose as a basis $\{|1,0\rangle,|0,1\rangle\}$. We are interested in the minimum of $\left\langle\Phi_{L}\left|\hat{n}_{L}\right| \Phi_{L}\right\rangle+\left\langle\Phi_{R}\left|\hat{n}_{R}\right| \Phi_{R}\right\rangle$, such that $\left\langle\Phi_{L} \mid \Phi_{R}\right\rangle=\gamma_{L R} \equiv \cos (\theta)$. We write these two wave functions as $\left|\Phi_{L}\right\rangle=\sin \left(\theta_{L}\right)|1,0\rangle+\cos \left(\theta_{L}\right)|0,1\rangle$ and $\left|\Phi_{R}\right\rangle=$ $\cos \left(\theta_{R}\right)|1,0\rangle+\sin \left(\theta_{R}\right)|0,1\rangle$. As a result of the corresponding minimization (7), the three angles are related as $\theta_{L}=\theta_{R}=$ $(\pi / 2-\theta) / 2$, and the universal functional is equal to

$$
\mathcal{F}_{2,2}(\theta)=2 \sin ^{2}\left(\frac{\pi}{4}-\frac{\theta}{2}\right)=1-\sin (\theta),
$$

which is one of the few analytical results for a universal functional that can be found in the literature $[49,50]$.

Machine learning. Despite the spectacular rise of machine learning in the study of quantum many-body systems, no implementation is known so far for the theory of reduced density matrices [51,52]. One of the reasons for this lack of progress is the large amount of constraints swarming in functional theories. We now discuss how our findings will facilitate learning the universal functional of bosonic systems. Notice that a more appealing way of writing the functional in Eq. (7) is the following: Let us choose a basis for the vector space $\mathcal{G}_{\gamma}$, say, $\left\{|\mathbf{m}\rangle \in \mathcal{H}_{N-1}\right\}$. A set of wave functions of the sort needed in the minimization (7) can now be written as $\left|\Phi_{j}\right\rangle=$ $\sum d_{j \mathbf{m}}|\mathbf{m}\rangle$. The condition of Eq. (4) reads $\mathbf{d d}^{\dagger}=\gamma$, where we have defined the matrix $[\mathbf{d}]_{j \mathbf{m}}=d_{j \mathbf{m}}$. Using the singular value decomposition for such a matrix, we have $\mathbf{d}=\mathbf{U} \boldsymbol{\Sigma} \mathbf{V}^{\dagger}$, with $\mathbf{U}$ and $\mathbf{V} M \times M$ unitary matrices [53,54]. Since $\boldsymbol{\Sigma} \boldsymbol{\Sigma}^{\dagger}=\mathbf{U}^{\dagger} \gamma \mathbf{U}$, it is now clear that the spectral decomposition of $\gamma$ is equal to $\Sigma \Sigma^{T}$. As a consequence, we obtain $[\Sigma]_{\alpha \alpha}=\sqrt{n_{\alpha}}$, where $\left\{n_{\alpha}\right\}$ are the eigenvalues of $\gamma$. Collecting these results, we obtain that the exact universal functional (7) can be explicitly written in terms of the eigenvalues and the eigenvectors of $\gamma$ (contained in the matrix $\mathbf{U}$ ):

$$
\mathcal{F}_{W}[\gamma]=\sum_{\alpha \beta} \sqrt{n_{\alpha} n_{\beta}} \Delta_{\alpha \beta}(\mathbf{U}, \mathbf{V}),
$$

where $\quad \Delta_{\alpha \beta}(\mathbf{U}, \mathbf{V})=\sum_{i, \mathbf{m m}^{\prime}} u_{i \alpha}^{*} u_{i \beta} v_{\mathbf{m} \alpha}^{*} v_{\mathbf{m}^{\prime} \beta}\left\langle\mathbf{m}^{\prime}\left|\hat{n}_{i}\right| \mathbf{m}\right\rangle$. The concrete form of the functional presented in Eq. (9) is striking: For fermionic density matrix functional theory, the square root of the occupation numbers in Eq. (9) is known to be the optimal choice for Ansätze of the form $n_{i}^{\alpha} n_{j}^{1-\alpha}$, compatible with the integral relation between the one- and two-body reduced density matrices [55-58], even for systems out of equilibrium [59]. As we can see, the only freedom in the functional (9) is the matrix $\mathbf{V}$, which is, unlike $\mathbf{U}$ and $n_{\alpha}$, not fixed by $\gamma$. We use this degree of freedom to introduce a standard optimization problem on a connected manifold $\mathcal{M}$ :

$$
\mathcal{F}_{N, M}[\gamma]=\min _{\mathbf{V} \in \mathcal{M}} \sum_{\alpha \beta} \sqrt{n_{\alpha} n_{\beta}} \Delta_{\alpha \beta}\left(\mathbf{U}_{\gamma}, \mathbf{V}\right),
$$

where we have included a subindex in $\mathbf{U}_{\gamma}$ to remember that such a matrix is defined by $\gamma$. Notice that the manifold $\mathcal{M}$ is essentially the set of special orthogonal matrices of dimension $M$, which generates the space $\mathcal{G}_{\gamma}$. Although the definition of such a space is far from trivial (and we will leave this question open for future research), it is possible to establish some elementary facts. For instance, in the strongly correlated regime $U / t \gg 1$ with integer filling factor $\alpha=N / M$, $\mathcal{G}_{\gamma}=\operatorname{span}\left\{b_{i}|\alpha, \ldots, \alpha\rangle\right\}$.

To make further progress on our problem, notice that optimization problems of the form $\min _{x \in \mathcal{M}} f(x)$ over a connected manifold $\mathcal{M}$ can be transformed into an unconstrained one of the form $\min _{y \in \mathbb{R}^{n}} f(\phi(y))$ by lifting the function $f$ to the current tangent space $T_{x} \mathcal{M}[60,61]$. The map $\phi: \mathbb{R}^{n} \rightarrow \mathcal{M}$ is called a trivialization map [60]. By letting the minimization in Eq. (10) run over the set of special orthogonal matrices in dimension $M$, the relevant minimization space turns out to be a Euclidian space $\mathbb{R}^{M}$. As a result, finding the universal functional of RDMFT presents itself as an unconstrained minimization problem. This is the crucial and last finding of this Research Letter, as it finally allows us to compute the universal bosonic functional by solving the problem directly over the set of orthonormal matrices.

Modern machine learning frameworks such as PYTORCH [62] provide fast and rather efficient ways of performing optimizations on connected manifolds of the type we consider here. For the results we will present below, we have implemented the constrained minimization (10) in PYTORCH with the constrained minimization toolkit GEOTORCH [63]. As a first step we implemented a minimization procedure where for each 1RDM the matrix $\mathbf{V}$ in Eq. (10) is optimized to produce the universal functional. As a second step we trained a neural network as the universal bosonic functional (see below).

Results. In Fig. 2 we present the results for the Bose-Hubbard model (6) for $M$ sites and $(\alpha M)$ bosons, for $M=2,4,6$ and $\alpha=1,2$. For this example, we have considered $\gamma$ of the form $\gamma_{i i}=\alpha$ and $\gamma_{i j}=\alpha \eta$, for $i \neq j$ with $0 \leqslant \eta \leqslant 1$ (this choice ensures the positive semidefiniteness of $\gamma$ [64]). The systems are fully condensated when $\eta=1$ (i.e., an occupation number is macroscopically populated). For comparison, all functionals have been normalized to 0 in the lower point (i.e., $\eta=0$ ) and to 1 in the upper point $(\eta=1)$. The exact known results for $M=2$ in Eq. (8) are verified in our calculations. Furthermore, we observe the existence of the Bose-Einstein force discovered in Ref. [21], generalized in Ref. [22], and proved in Ref. [65], namely, the divergence of the gradient $\partial_{\eta} \mathcal{F}_{N, M}(\eta) \rightarrow\left(N-N_{\mathrm{BEC}}\right)^{\zeta}$, with $\zeta<0$, when approaching to the condensation point (i.e., $\eta \rightarrow 1)$. The striking similarities of the functionals $\mathcal{F}_{M, M}[\gamma]$ and $\mathcal{F}_{2 M, M}[\gamma]$ suggest the existence of a universal functional independent of $\alpha$, up to appropriate normalizations.

In functional theories the knowledge of the functional's form is as important as the knowledge of its derivative, as 


$$
\alpha=1
$$

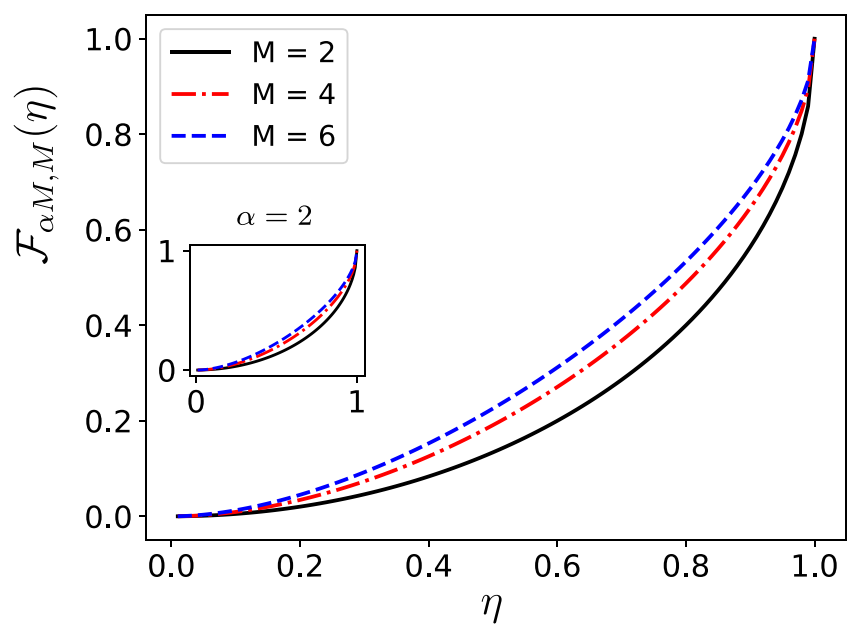

FIG. 2. Universal functionals $\mathcal{F}_{M, M}[\gamma]$ and $\mathcal{F}_{2 M, M}[\gamma]$ for the $M$-site one-dimensional (1D) Bose-Hubbard model with filling factor $\alpha=1,2$, for $M=2,4,6$ sites (see text). The functional corresponds to all $1 \mathrm{RDM}$ with $\gamma_{i i}=\alpha=N / M$ and $\gamma_{i j}=N \eta / M$ for $i \neq j$. All functionals are convex, as expected. For easy comparison, all functionals have been normalized to 0 in the lower point $(\eta=0)$ and to 1 in the upper point $(\eta=1)$.

both are needed for a ground-state calculation. To perform the derivative of the functional, we trained a neural network to output the matrix $\mathbf{V}$ using the degrees of freedom of our $1 \mathrm{RDM}$ as input. This has multiple advantages. First, once the functional is trained for given particle and site numbers, it can be evaluated for any $\gamma$. Secondly, the automatic differentiation allows an exact evaluation of the gradient $\nabla_{\gamma} \mathcal{F}_{N, M}[\gamma]$ without further work. For the Bose-Hubbard dimer it was sufficient to use the diagonal term $\eta=\gamma_{i(i+1)}$ and its square as inputs. The calculation was structured as follows:

$$
\operatorname{FCNN}_{N, M, \theta}\left(\eta, \eta^{2}, \mathbf{U S}\right) \rightarrow \mathbf{V} \rightarrow \mathcal{F}_{N, M, \theta}[\gamma] .
$$

Here, $\mathrm{FCNN}_{N, M, \theta}$ is a fully connected network for $N$ particles and $M$ sites with the parameters $\theta$ and

$$
\mathbf{U S}=\mathbf{U} \times\left(\begin{array}{cccc}
n_{0} & 0 & \cdots & 0 \\
0 & n_{1} & \cdots & 0 \\
\vdots & \vdots & \ddots & \vdots \\
0 & 0 & 0 & n_{M}
\end{array}\right)
$$

calculated from the eigenvectors $\mathbf{U}$ and eigenvalues $n_{i}$ of $\gamma$. During training we minimize the functional $\mathcal{F}_{N, M, \theta}[\gamma]$ for a set of $\gamma$ in parallel. The networks used two hidden layers, with exponential linear units (ELU) [66] as activation functions, and the output of the last layer was used to create an orthogonal matrix through a matrix exponential. The network for the dimer was trained on the set $\eta \in(0.005,0.001, \ldots, 0.995)$. When evaluating on the set $(0.0025,0.0075, \ldots, 0.9925)$, the maximum absolute error is smaller than $10^{-15}$. The network for $N=4, M=4$ was trained on the same set. Remarkably, as shown in Fig. 3, for the Bose-Hubbard dimer (for which we can compare with exact results) the derivatives provided by the neural network only deviate by $\sim 10^{-13}$ from the exact results.

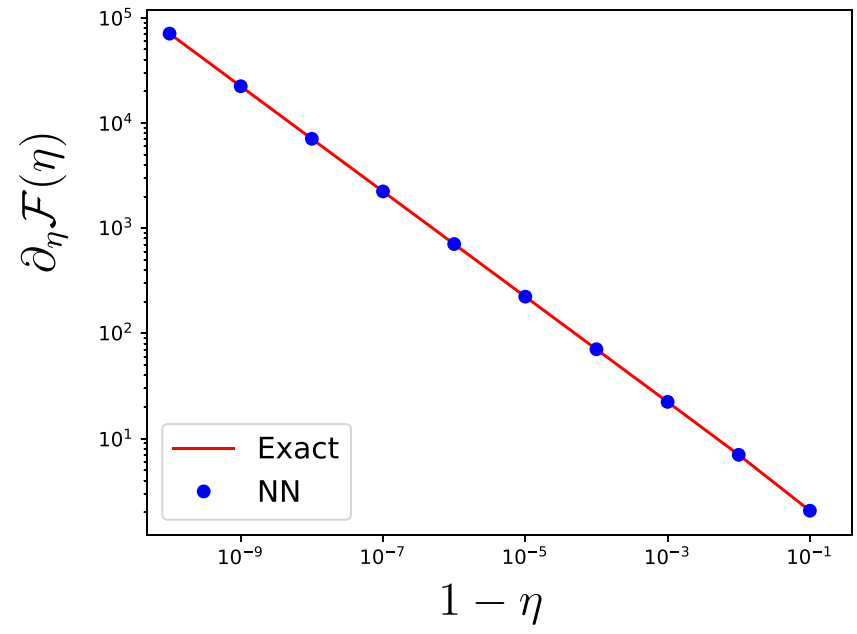

FIG. 3. Comparison between the gradient of the functional for the Bose-Hubbard dimer computed with the neural network (NN) and the exact analytical results as a function of $1-\eta$ (see text). Notice the log-log scale.

We demonstrate now that our approach allows us to compute the ground-state energy and 1RDM for a large system. Notice first that for a fixed filling factor $\alpha=N / M$, the energy of the ground state of the Bose-Hubbard model (6) can be computed as the minimum of the energy functional $\mathcal{E}_{N, M}[\gamma]=$ $-2 t \sum_{i} \gamma_{i(i+1)}+\mathcal{F}_{N, M}[\gamma]$. By performing the minimization $\nabla_{\gamma} \mathcal{E}_{N, M}[\gamma]=0$ on the domain of positive semidefinite matrices, it is then possible to compute the ground-state energy of the system. To generate the functional in that domain, we have optimized the Ansatz $\gamma_{i j}=\eta^{\kappa} \alpha(0 \leqslant \eta \leqslant 1)$ with $2 \leqslant \kappa \leqslant 8$, for $|j-i|>1$, with $\eta=\gamma_{i(i+1)}$. This is motivated by the fact that when $U / t \gg 0, \gamma_{i j} \approx 0, \forall i>j$, and when $U / t \ll$ $1 \gamma_{i j} \approx \alpha, \forall i j$. Following this prescription, we have computed the ground-state energy for the 40-site Bose-Hubbard model with 40 bosons. The dimension of the corresponding Hilbert space, being of the order of $5.3 \times 10^{22}$, is out of reach for exact diagonalization and prohibits performing the exact constrained search approach. To solve this problem, we have (i) chosen for the space $\mathcal{G}_{\gamma}$, the subspace generated by the kets $\left|\Phi_{i}\right\rangle=\hat{b}_{i}|\Psi\rangle$, by choosing $|\Psi\rangle=|1, \ldots, 1\rangle$ (RDMFT1), and (ii) used the exact functional of the dimer (8), appropriately rescaled (RDMFT2). The energy predictions of our machine learning functionals are quite remarkable, given the subspaces we have chosen. Indeed, the results presented in Fig. 4(a) indicate that the predicted RDMFT results are in good agreement with the QMC energies: The errors around $U / t=4$ are only due to the approximation of the space $\mathcal{G}_{\gamma}$. In order to check the quality of the approximate functionals further, we have also plotted the relative error in Fig. 4(b). We observe that this error is below $8 \%$ and practically zero for large and weak interaction. In addition, notice that our implementation is able to approximate the whole range of energies, not only the weakly (the sector easily described by Bogoliubov methods) or the strongly correlated regimes.

Conclusion. We have demonstrated the viability of approximating universal bosonic functionals in a quite efficient way. The main ingredient of the computation is a simplification of the constrained search approach that we have introduced in 

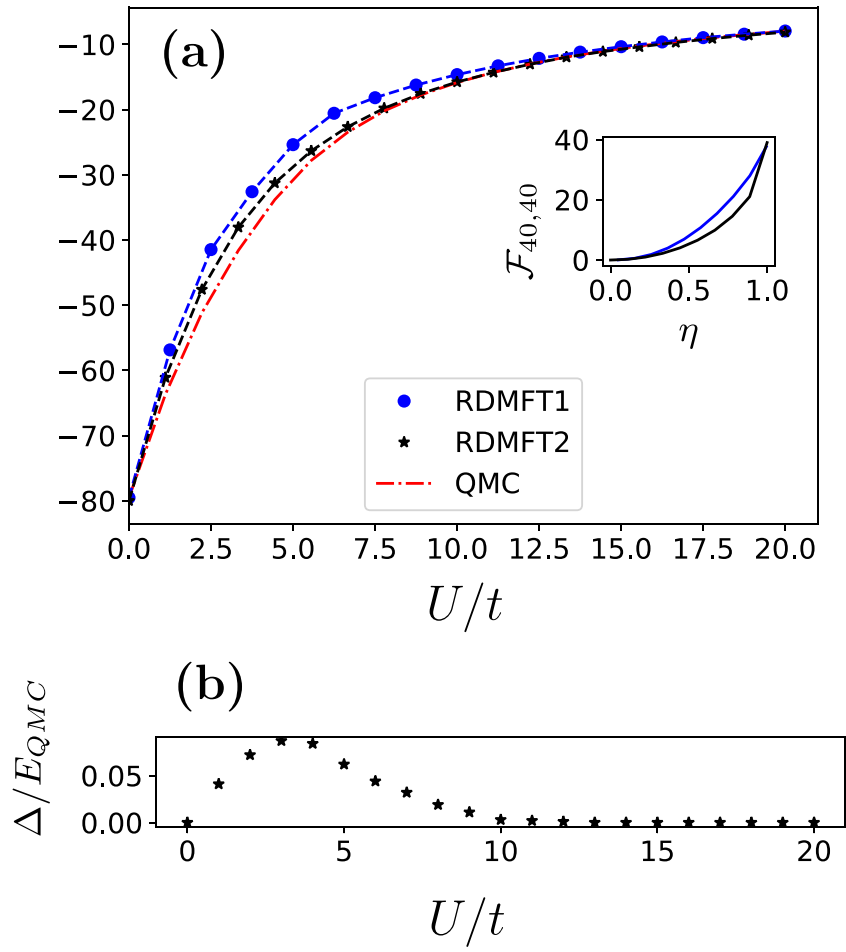

FIG. 4. Ground-state energy of the 40-site 1D Bose-Hubbard model with 40 bosons. In (a) the energy is plotted as a function of the relative strength $U / t$. In blue are the results computed using the functional (10) (RDMFT1) and the Ansatz $\left|\Phi_{i}\right\rangle=b_{i}|1, \ldots, 1\rangle$. In black we use as an Ansatz, for the functional the exact expression of the dimer appropriately rescaled (RDMFT2). In red are the results computed with the QMC method. In the inset in (a) the functionals $\mathcal{F}_{N, N}(\eta)$ are plotted as a function of $\eta$, the nearest-neighbor offdiagonal value of the 1RDM. In (b) the relative errors $\Delta / E_{\mathrm{QMC}}$, where $\Delta=E_{\mathrm{RDMFT} 2}-E_{\mathrm{QMC}}$, are plotted as a function of $U / t$. this Research Letter based on the Schmidt decomposition of the wave function. This formulation of reduced density matrix functional theory (RDMFT) speeds up the design of reliable approximations for the universal functionals for systems with translational symmetry. The quality of the numerical results obtained in this Research Letter highlights the potential of RDMFT to become a competitive tool for computing properties of bosonic ground states with high-dimensional Hilbert spaces. Strikingly, since RDMFT takes into account the whole range of bosonic correlations and does not present dimensional or sign problems, it offers a range of new possibilities. For instance, frustrated bosonic systems can be studied in a direct manner [67]. Bosonic systems with impurities, composites of ultracold atoms, or even superconducting systems [68-71] can also potentially be addressed within this framework. As an outlook of this work, we leave open a new line of research based on extending our findings to systems with internal degrees of freedom, finite temperatures, or broken symmetries. We also expect that previous works in the context of a two-body reduced density matrix [72,73] will also benefit from our approach.

All codes to reproduce, examine, and improve our proposed analysis are freely available online [74].

Acknowledgments. We thank Jonathan Siegel, Matt Eiles, Adam Sawicki, Rolf Schilling, Joe Varilly, and Jakob Wolff for helpful discussions. We are most grateful to Peter Karpov for constructive feedback and insight and for providing us the QMC energies of the Bose-Hubbard model. M.F. was partially supported by the Research Fund of the University of Basel for Excellent Junior Researchers. C.L.B.-R. was supported by the MPI-PKS through a next-step fellowship.
[1] P. Hohenberg and W. Kohn, Inhomogeneous electron gas, Phys. Rev. 136, B864 (1964).

[2] R. O. Jones, Density functional theory: Its origins, rise to prominence, and future, Rev. Mod. Phys. 87, 897 (2015).

[3] M. A. Cazalilla, R. Citro, T. Giamarchi, E. Orignac, and M. Rigol, One dimensional bosons: From condensed matter systems to ultracold gases, Rev. Mod. Phys. 83, 1405 (2011).

[4] B. Chatterjee, C. Lévêque, J. Schmiedmayer, and A. U. J. Lode, Detecting One-Dimensional Dipolar Bosonic Crystal Orders via Full Distribution Functions, Phys. Rev. Lett. 125, 093602 (2020)

[5] D. González-Cuadra, P. R. Grzybowski, A. Dauphin, and M. Lewenstein, Strongly Correlated Bosons on a Dynamical Lattice, Phys. Rev. Lett. 121, 090402 (2018).

[6] M. P. A. Fisher, P. B. Weichman, G. Grinstein, and D. S. Fisher, Boson localization and the superfluid-insulator transition, Phys. Rev. B 40, 546 (1989).

[7] L. P. Pitaevskii and S. Stringari, Bose-Einstein Condensation (Clarendon, Oxford, 2003).

[8] F. Dalfovo, S. Giorgini, L. Pitaevskii, and S. Stringari, Theory of Bose-Einstein condensation in trapped gases, Rev. Mod. Phys. 71, 463 (1999).
[9] J. Gubernatis, N. Kawashima, and P. Werner, Quantum Monte Carlo Methods: Algorithms for Lattice Models (Cambridge University Press, Cambridge, 2016).

[10] H. Saito, Solving the Bose-Hubbard model with machine learning, J. Phys. Soc. Jpn. 86, 093001 (2017).

[11] K. Choo, G. Carleo, N. Regnault, and T. Neupert, Symmetries and Many-Body Excitations with Neural-Network Quantum States, Phys. Rev. Lett. 121, 167204 (2018).

[12] I. Bloch, J. Dalibard, and W. Zwerger, Many-body physics with ultracold gases, Rev. Mod. Phys. 80, 885 (2008).

[13] C. Chin, R. Grimm, P. Julienne, and E. Tiesinga, Feshbach resonances in ultracold gases, Rev. Mod. Phys. 82, 1225 (2010).

[14] I. Bloch, Ultracold quantum gases in optical lattices, Nat. Phys. 1, 23 (2005).

[15] C. Gross and I. Bloch, Quantum simulations with ultracold atoms in optical lattices, Science 357, 995 (2017).

[16] F. Schäfer, T. Fukuhara, S. Sugawa, Y. Takasu, and Y. Takahashi, Tools for quantum simulation with ultracold atoms in optical lattices, Nat. Rev. Phys. 2, 411 (2020).

[17] R. Schmied, J.-D. Bancal, B. Allard, M. Fadel, V. Scarani, P. Treutlein, and N. Sangouard, Bell correlations in a BoseEinstein condensate, Science 352, 441 (2016). 
[18] M. Fadel, T. Zibold, B. Décamps, and P. Treutlein, Spatial entanglement patterns and Einstein-Podolsky-Rosen steering in Bose-Einstein condensates, Science 360, 409 (2018).

[19] P. Kunkel, M. Prüfer, H. Strobel, D. Linnemann, A. Frölian, T. Gasenzer, M. Gärttner, and M. K. Oberthaler, Spatially distributed multipartite entanglement enables EPR steering of atomic clouds, Science 360, 413 (2018).

[20] K. Lange, J. Peise, B. Lücke, I. Kruse, G. Vitagliano, I. Apellaniz, M. Kleinmann, G. Tóth, and C. Klempt, Entanglement between two spatially separated atomic modes, Science 360, 416 (2018).

[21] C. L. Benavides-Riveros, J. Wolff, M. A. L. Marques, and C. Schilling, Reduced Density Matrix Functional Theory for Bosons, Phys. Rev. Lett. 124, 180603 (2020).

[22] J. Liebert and C. Schilling, Functional theory for Bose-Einstein condensates, Phys. Rev. Res. 3, 013282 (2021).

[23] T. Gilbert, Hohenberg-Kohn theorem for nonlocal external potentials, Phys. Rev. B 12, 2111 (1975).

[24] K. Pernal and K. J. H. Giesbertz, Reduced density matrix functional theory (RDMFT) and linear response time-dependent RDMFT, Top. Curr. Chem. 368, 125 (2016).

[25] K. Sakmann, A. I. Streltsov, O. E. Alon, and L. S. Cederbaum, Reduced density matrices and coherence of trapped interacting bosons, Phys. Rev. A 78, 023615 (2008).

[26] A. Bulgac and S. Jin, Dynamics of Fragmented Condensates and Macroscopic Entanglement, Phys. Rev. Lett. 119, 052501 (2017).

[27] A. Aloy, M. Fadel, and J. Tura, The quantum marginal problem for symmetric states: applications to variational optimization, nonlocality and self-testing, New J. Phys. 23, 033026 (2021).

[28] M. Walter, B. Doran, D. Gross, and M. Christandl, Entanglement polytopes: Multiparticle entanglement from singleparticle information, Science 340, 1205 (2013).

[29] A. Sawicki, M. Oszmaniec, and M. Kuś, Convexity of momentum map, Morse index, and quantum entanglement, Rev. Math. Phys. 26, 1450004 (2014).

[30] A. Sawicki, M. Oszmaniec, and M. Kuś, Critical sets of the total variance can detect all stochastic local operations and classical communication classes of multiparticle entanglement, Phys. Rev. A 86, 040304(R) (2012).

[31] X.-D. Yu, T. Simnacher, N. Wyderka, H. C. Nguyen, and O. Gühne, A complete hierarchy for the pure state marginal problem in quantum mechanics, Nat. Commun. 12, 1012 (2021).

[32] B. Kalita, L. Li, R. J. McCarty, and K. Burke, Learning to approximate density functionals, Acc. Chem. Res. 54, 818 (2021).

[33] F. Brockherde, L. Vogt, L. Li, M. Tuckerman, K. Burke, and K.R. Müller, Bypassing the Kohn-Sham equations with machine learning, Nat. Commun. 8, 872 (2017).

[34] J. Schmidt, C. L. Benavides-Riveros, and M. A. L. Marques, Machine learning the physical nonlocal exchange-correlation functional of density-functional theory, J. Phys. Chem. Lett. 10, 6425 (2019).

[35] L. Li, S. Hoyer, R. Pederson, R. Sun, E. D. Cubuk, P. Riley, and K. Burke, Kohn-Sham Equations as Regularizer: Building Prior Knowledge into Machine-Learned Physics, Phys. Rev. Lett. 126, 036401 (2021).

[36] J. T. Margraf and K. Reuter, Pure non-local machinelearned density functional theory for electron correlation, Nat. Commun. 12, 344 (2021).
[37] J. R. Moreno, G. Carleo, and A. Georges, Deep Learning the Hohenberg-Kohn Maps of Density Functional Theory, Phys. Rev. Lett. 125, 076402 (2020).

[38] D. Gibney, J.-N. Boyn, and D. Mazziotti, Toward a resolution of the static Ccorrelation problem in density functional theory from semidefinite programming, J. Phys. Chem. Lett. 12, 385 (2021).

[39] M. Levy, Universal variational functionals of electron densities, first-order density matrices, and natural spin-orbitals and solution of the $v$-representability problem, Proc. Natl. Acad. Sci. USA 76, 6062 (1979).

[40] C. Schilling, C. L. Benavides-Riveros, A. Lopes, T. Maciażek, and A. Sawicki, Implications of pinned occupation numbers for natural orbital expansions: I. Generalizing the concept of active spaces, New J. Phys. 22, 023001 (2020).

[41] P.-O. Löwdin and H. Shull, Natural Orbitals in the Quantum Theory of Two-Electron Systems, Phys. Rev. 101, 1730 (1956).

[42] C. Schilling and R. Schilling, Diverging Exchange Force and Form of the Exact Density Matrix Functional, Phys. Rev. Lett. 122, 013001 (2019).

[43] K. J. H. Giesbertz, Implications of the unitary invariance and symmetry restrictions on the development of proper approximate one-body reduced-density-matrix functionals, Phys. Rev. A 102, 052814 (2020).

[44] M. Fadel, A. Aloy, and J. Tura, Bounding the fidelity of quantum many-body states from partial information, Phys. Rev. A 102, 020401(R) (2020).

[45] P. Mori-Sánchez and A. J. Cohen, Exact density functional obtained via the Levy constrained search, J. Phys. Chem. Lett. 9, 4910 (2018).

[46] K. J. H. Giesbertz and M. Ruggenthaler, One-body reduced density-matrix functional theory in finite basis sets at elevated temperatures, Phys. Rep. 806, 1 (2019).

[47] D. Jaksch, C. Bruder, J. I. Cirac, C. W. Gardiner, and P. Zoller, Cold Bosonic Atoms in Optical Lattices, Phys. Rev. Lett. 81, 3108 (1998).

[48] See Supplemental Material at http://link.aps.org/supplemental/ 10.1103/PhysRevResearch.3.L032063 for technical details on the presentation of the unconstrained search approach for translational invariant systems, the solution of the Hubbard dimer within this framework, and technical details on the neural networks.

[49] A. J. Cohen and P. Mori-Sánchez, Landscape of an exact energy functional, Phys. Rev. A 93, 042511 (2016).

[50] W. Töws and G. M. Pastor, Lattice density functional theory of the single-impurity Anderson model: Development and applications, Phys. Rev. B 83, 235101 (2011).

[51] G. Carleo, I. Cirac, K. Cranmer, L. Daudet, M. Schuld, N. Tishby, L. Vogt-Maranto, and L. Zdeborová, Machine learning and the physical sciences, Rev. Mod. Phys. 91, 045002 (2019).

[52] J. Carrasquilla, Machine learning for quantum matter, Adv. Phys.X 5, 1797528 (2020).

[53] N. Gigena, M. Di Tullio, and R. Rossignoli, One-body entanglement as a quantum resource in fermionic systems, Phys. Rev. A 102, 042410 (2020).

[54] R. Lo Franco and G. Compagno, Indistinguishability of Elementary Systems as a Resource for Quantum Information Processing, Phys. Rev. Lett. 120, 240403 (2018). 
[55] A. M. K. Müller, Explicit approximate relation between reduced two- and one-particle density matrices, Phys. Lett. A 105, 446 (1984).

[56] M. Buijse and E. Baerends, An approximate exchangecorrelation hole density as a functional of the natural orbitals, Mol. Phys. 100, 401 (2002).

[57] R. L. Frank, E. H. Lieb, R. Seiringer, and H. Siedentop, Müller's exchange-correlation energy in density-matrix-functional theory, Phys. Rev. A 76, 052517 (2007).

[58] C. L. Benavides-Riveros and M. A. L. Marques, Static correlated functionals for reduced density matrix functional theory, Eur. Phys. J. B 91, 133 (2018).

[59] C. L. Benavides-Riveros and M. A. L. Marques, On the time evolution of fermionic occupation numbers, J. Chem. Phys. 151, 044112 (2019).

[60] M. Lezcano-Casado, Trivializations for gradient-based optimization on manifolds, in Advances in Neural Information Processing Systems, NeurIPS (Curran Associates, Red Hook, NY, 2019), pp. 9154-9164.

[61] J. Siegel, Accelerated optimization with orthogonality constraints, J. Comput. Math. 39, 207 (2020).

[62] A. Paszke, S. Gross, S. Chintala, G. Chanan, E. Yang, Z. DeVito, Z. Lin, A. Desmaison, L. Antiga, and A. Lerer, Automatic differentiation in pytorch, in NIPS 2017 AutodiffWorkshop: The Future of Gradient-based Machine Learning Software and Techniques (2017).

[63] GEOTORCH, https://github.com/Lezcano/geotorch.

[64] E. H. Lieb and R. Seiringer, The Stability of Matter in Quantum Mechanics (Cambridge University Press, Cambridge, 2009).
[65] T. Maciażek, Repulsively diverging gradient of the density functional in the reduced density matrix functional theory, arXiv:2103.17069.

[66] D.-A. Clevert, T. Unterthiner, and S. Hochreiter, Fast and accurate deep network learning by exponential linear units (ELUs), arXiv:1511.07289.

[67] F. Wang, F. Pollmann, and A. Vishwanath, Extended Supersolid Phase of Frustrated Hard-Core Bosons on a Triangular Lattice, Phys. Rev. Lett. 102, 017203 (2009).

[68] A. L. Hunter, M. T. Eiles, A. Eisfeld, and J. M. Rost, Rydberg Composites, Phys. Rev. X 10, 031046 (2020).

[69] J. Schmidt, C. L. Benavides-Riveros, and M. A. L. Marques, Representability problem of density functional theory for superconductors, Phys. Rev. B 99, 024502 (2019).

[70] J. Schmidt, C. L. Benavides-Riveros, and M. A. L. Marques, Reduced density matrix functional theory for superconductors, Phys. Rev. B 99, 224502 (2019).

[71] O. E. Alon, A. I. Streltsov, and L. S. Cederbaum, Zoo of Quantum Phases and Excitations of Cold Bosonic Atoms in Optical Lattices, Phys. Rev. Lett. 95, 030405 (2005).

[72] D. A. Mazziotti and D. R. Herschbach, Boson Correlation Energies from Reduced Hamiltonian Interpolation, Phys. Rev. Lett. 83, 5185 (1999).

[73] G. Gidofalvi and D. A. Mazziotti, Boson correlation energies via variational minimization with the two-particle reduced density matrix: Exact $N$-representability conditions for harmonic interactions, Phys. Rev. A 69, 042511 (2004).

[74] https://github.com/cabenav/Machine-Learning-UniversalBosonic-Functionals. 